\title{
Ultrasonic Evaluation in Infants with Intracranial Lesions
}

\author{
TAKASHI HAYASHI*, AKIRA ISHIBASHI*, TAKEO HASHIMOTO**, \\ KOHICHI EGAMI*** AND SHINKEN KURAMOTO****
}

Department of Neurosurgery*, Department of Neonatology** and Department ot Radiology,

St. Mary's Hospital ${ }^{* * *}$, Kurume, 830 and Department of Neurosurgery, Kurume University School of Medicine****, Kurume, 830 Japan

Received for publication February 7, 1981

\begin{abstract}
Key words: Ultrasonography - Ultrasound ventriculography - B-modescanning - CT scan - Hydrocephalus - Falx cerebri.
\end{abstract}

Computerized tomography (CT) has greatly increased both the accuracy and ease with which intracranial lesions are diagnosed. However, when repeated examination is required, the effects of radiation exposure cannot be ignored (Bhave et al., 1977). Accordingly it is necessary to select the diagnostic procedure which minimizes radiation exposure. Diagnostic ultrasound apparatus has been actively investigated to adress this problem. Occasional reports on the investigation of this approach have appeared (Kossoff et al, 1974; Garrett et al., 1975; Shkolnik et al., 1979; Haber et al., 1980).

We used B-mode autocompound scan (U.I. Ocotoson) in the diagnosis of various intracranial pathological states in the newborn, and we compared the results with those of the CT scanner. In this paper we present a summary of the advantages and disadvantages of the ultrasound apparatus.

\section{Advantages}

1) There is no significant difference between the results obtained from this apparatus and those obtained from the CT scanner with respect to the resolving capacity of the cerebral ventricle.

2) There is no exposure to x-ray irradiation.

3) The use of a tranquilizer for these patients is not required, because examination is extremely rapid.

4) The resolving capacity of ultrasound apparatus is greater than that of the CT scanner. It is particularly useful with respect to the midline structure of the septum pellucidum and the falx cerebri, and in discerning the alobar holoprosencephaly and the hydranencephaly. It also has better resolving capacity for porencephaly, which is often seen in postmeningitic hydrocephalus and multiloculated ventricle by septum formation in the brain.

\section{Disadvantages}

1) The CT scanner can indicate the intensity of components in units of Hounsfield's numbers, a feature not available with ultrasound apparatus at the present stage.

Correspondence address: Takashi Hayashi, M. D.

Department of Neurosurgery, St. Mary’s Hospital, 422 Honmachi Tsubuku Kurume, 830 Japan. 


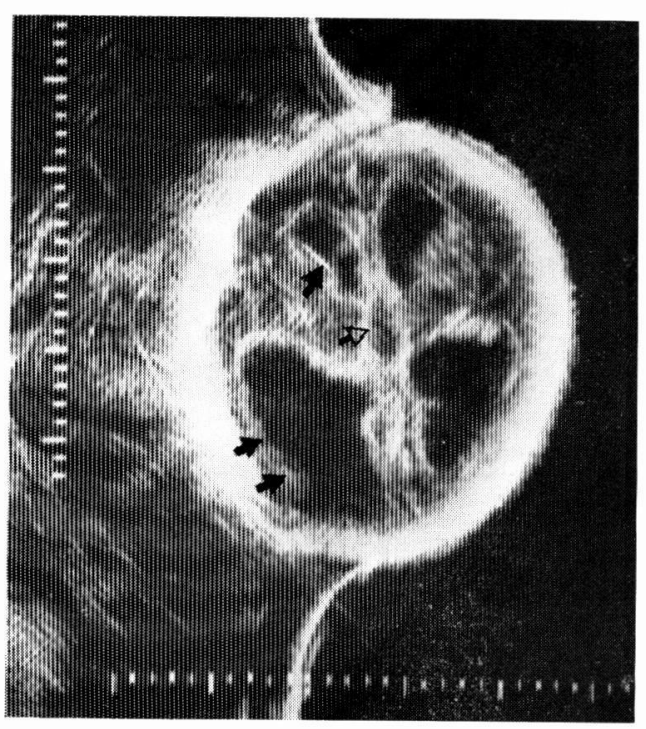

Fig. 1. Ultrasound of hydrocephalic infant due to intraventricular hemorrhage. Ventriculomegaly can be seen clearly. Closed arrow: frontal horn of the lateral ventricle. Open arrow: third ventricle. Two closed arrow: posterior horn of the lateral ventricle.
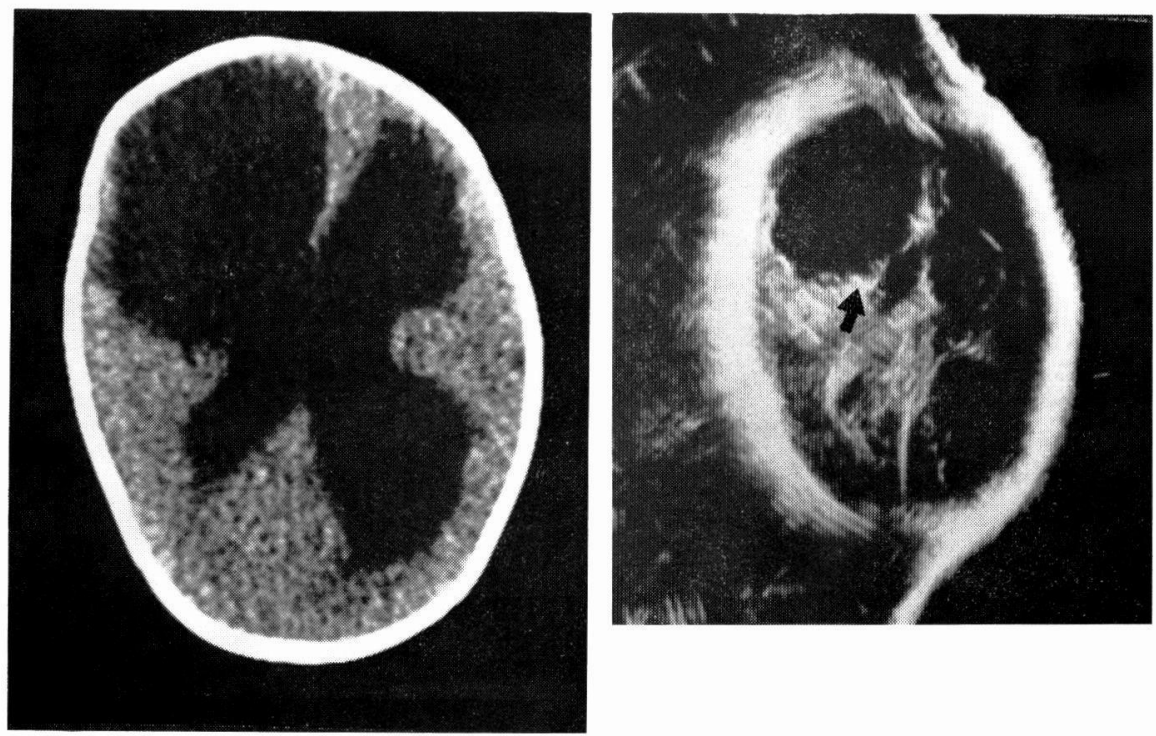

Fig. 3. Postmeningitis hydrocephalus: Left: CT scan. Right: ultrasound. Porncephalic cyst cannot be seen in CT scan, while it can be seen in ultrasound (arrow).

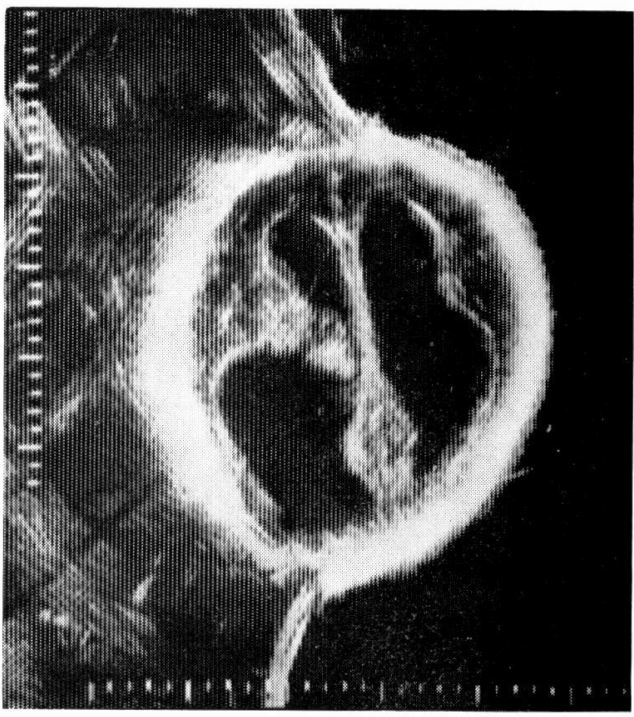

Fig. 2. Seme case as Fig. 1. This is $1 \mathrm{~cm}$ above the slice in Fig. 1. Falx cerebri and ventricular wall can be seen clealy. 
2) Since apparatus for bedside testing is not available, examination of premature infants or of newborns with high risk of mortality is more hazardous. For instance, it is necessary to remove the infant from the respirator. Body temperature may also decrease. This disadvantage, of course, also applies to the CT scanner.

3) The ultrasound apparatus described here does not have the same resolving capacity as does the CT scanner.

4) It is somewhat difficult to apply ultrasound technology to infants whose skull are thicker than normal or to those who have excessive amounts of hair.

\section{References}

Bhave, D. G., Kelsey, C. A. and Burnstein, J. (1871). Scattered radiation doses to infants and children during EMI head scans. Radi- ology, 124, 379-380.

Garrett, W. J., Kossoff, G. and Jones, R.F.C (1975). Ultrasonic cross-sectional visualization of hydrocephalus in infants. Neuroradiology, 8, 279-288.

Haber, K., Wachter, R. D., Christenson, P. C., VAucher, Y., SAHN, D. J, and Smith, R. (1980). Ultrasonic evaluation of intracranial pathology in infants: A new technique. Radiology, 134, $173-178$.

Kossoff, G., Garrett, W. J. and Radavanovich, G. (1974). Ultrasonic atlas of normal brain of infant. Ultrasound Med. Biol., 1, 259266.

Shkolnik, A. (1975). B-mode scanning of the infant brain. A new approach. Case report. craniopharyngioma. J. Clin. Ultrasound, 3 , $229-231$.

Skolnik, M. L., Rosenbaum, A.E. and Matzuk, T. (1979). Detection of dilated cerebral ventricles in infants: a correlation study between ulytrasound and computed tomography. Radiology, 131, 447-451. 